\title{
Untersuchung der Beanspruchung und des Zugkraftverlaufs raumgängiger Gleitketten
}

\author{
Dr.-Ing. P. Auerbach \\ Prof. Dr.-Ing. Klaus Nendel \\ TU Chemnitz, Professur Fördertechnik
}

Darstellung eines Belastungsmodells sowie einer FEM-Analyse der Spannungsverteilung in seitenbeweglichen Gleitketten beim Durchlaufen räumlicher Förderstrecken. Beschreibung der Ergebnisse von Messungen des Zugkraftverlaufs unter Variation belastungsrelevanter Parameter. Herleitung genauerer Gleichungen für die Kettenzugkraft in Gleitbögen und deren Abgleich mit gemessenen Kraftverläufen.

\section{Einleitung}

Fördersysteme auf Basis raumgängiger Kunststoff-Gleitketten werden in vielen Branchen zum Transport leichter Stückgüter (bis ca. $20 \mathrm{~kg}$ ) eingesetzt. Zu ihren Vorteilen zählen der schlupffreie, formschlüssige Antrieb, die Möglichkeit zum sauberen, schmierungsfreien Betrieb und die für horizontale und vertikale Kurven notwendige Seitenbeweglichkeit bzw. Rückbiegewilligkeit der Ketten. Der Aufbau der Fördersysteme aus Modulen, die sich weitgehend frei miteinander kombinieren lassen, ermöglicht vielfältige, räumliche Streckenlayouts.

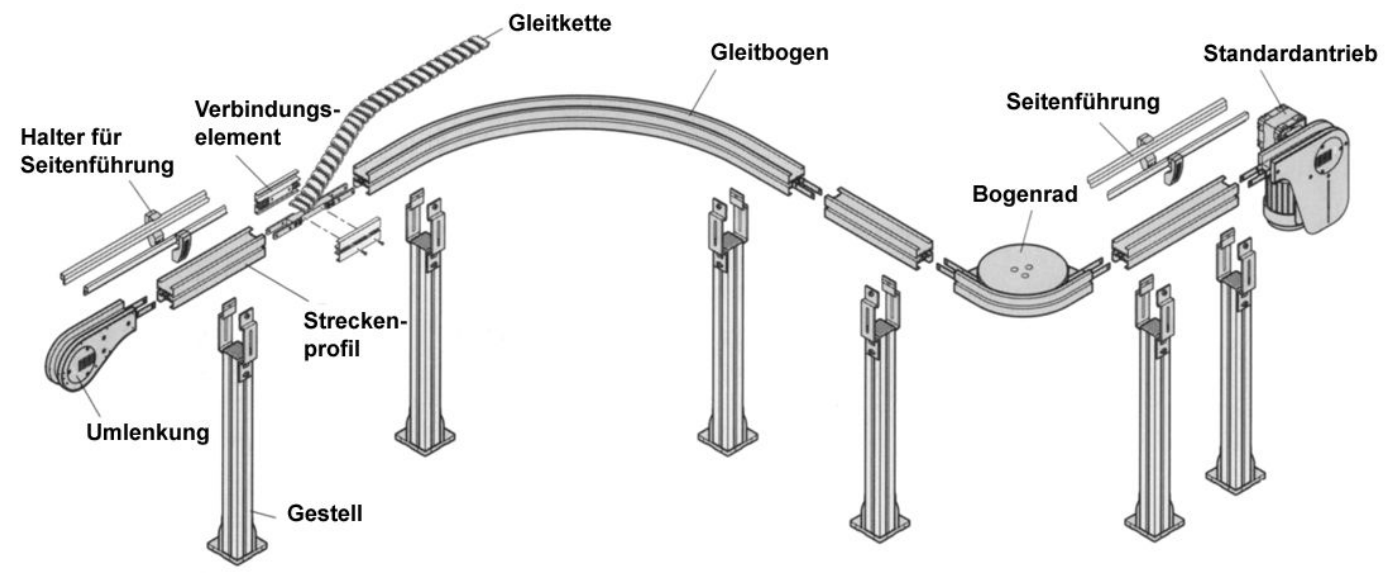

Abb. 1: Komponenten eines Gleitketten-Fördersystems nach [Bosch04]

Im Gegensatz zur weiten Verbreitung dieser Fördersysteme stehen die lückenhaften Kenntnisse über deren Beanspruchung, Haltbarkeit und Dimensionierung. In der einschlägigen Literatur sind dazu praktisch keine Angaben zu finden. Die in den Firmenschriften der Kettenhersteller angegebenen, vereinfachten Gleichungen erlauben nur eine überschlägige Auslegung der Ketten, die insbesondere keine Aussage über deren Lebensdauer liefern kann. Als Festigkeitskenngröße wird lediglich die statische Bruchkraft einer Kette herangezogen und durch einen Sicherheitsfaktor zwischen drei und vier geteilt. In Dauerversuchen wurde festgestellt, dass bei Ausnutzung der auf diese Weise berechneten zulässigen Zugkraft die Gleitketten je nach Streckenführung nur eine sehr geringe Lebensdauer (z.B. unter 500 h) erreichen. Darüber hinaus wurde festgestellt, dass die erreichte Lebensdauer stark davon abhängt, wieviele Kurven eine Förderstrecke enthält.

Um ein besseres Verständnis der Belastungen und Festigkeitseigenschaften von KunststoffGleitketten zu erzielen, wurden an der Professur Fördertechnik der TU Chemnitz verschiedene Untersuchungen auf beiden Gebieten durchgeführt, die in zwei aufeinander folgenden Artikeln veröffentlicht werden. Im vorliegenden Artikel werden die Ergebnisse dargestellt, die bei der Analyse der Belastungen und der Messung sowie genaueren mathematischen Beschreibung von Kettenzugkräften erzielt wurden. Eine weitere Veröffentlichung beschäftigt sich mit der Ermittlung von Dauerfestigkeitswerten von Kunststoff-Gleitketten und schlägt einen Ansatz vor, der erstmals eine Abschätzung der Lebensdauer, bezogen auf einen Kettenbruch, erlaubt. Weiterhin wird darin ein auf Grundlage der gewonnenen Erkenntnisse implementiertes Berechnungsprogramm vorgestellt, das eine schnelle und zuverlässige Auslegung von Gleitketten-Fördersystemen erlaubt. 


\section{Belastungsmodell}

Im Unterschied $\mathrm{zu}$ anderen Arten von Ketten bzw. Zugmitteln können die seitenbeweglichen Gleitketten nicht nur vertikal, sondern auch horizontal umgelenkt werden. Um besser verstehen zu können, welche Beanspruchung der Kettenglieder die verschiedenen Komponenten zur Streckenführung (Gerade, Antrieb, horizontale und vertikale Kurven etc.) hervorrufen, wurde für jede Komponente ein Belastungsmodell aufgestellt. In dieses räumliche Modell wurden alle im betrachteten Streckenabschnitt auf ein Kettenglied einwirkenden Kräfte eingetragen. Die Abbildungen 2 und 3 zeigen dies am Beispiel der Modelle für Geraden und horizontale Kurven.

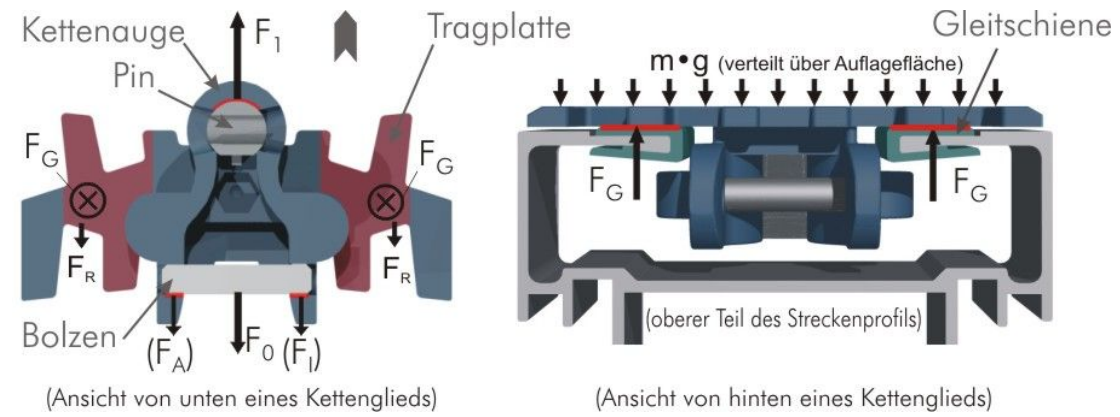

Abb. 2: Belastungsmodell für geraden Streckenabschnitt

Es wird erkennbar, dass eine Vielzahl von räumlich verteilten Kräften auf die Kettenglieder einwirken, die sich aber größenmäßig deutlich unterscheiden. Durch Beispielrechnungen mit typischen Parametern für Reibwerte, Gutmassen und -abmessungen sowie spezifische Kettenmasse wurde unterstrichen, dass die Kettenzugkraft $\left(F_{1}, F_{0}\right)$ die mit Abstand größte Kraft darstellt. Die weiteren Kräfte, die hauptsächlich durch die Gewichtskräfte von Fördergut (verteilt über die Auflagfläche des Gutes auf der Tragplatte) und Kette sowie durch Reibung an verschiedenen Kontaktstellen zum Führungssystem hervorgerufen werden, sind gegenüber der Kettenzugkraft vernachlässigbar klein. Dies lässt sich auch damit begründen, dass die (maximale) Kettenzugkraft die Summe vieler vergleichsweise kleiner Reibkräfte an allen Kettengliedern im Führungssystem darstellt. Man kann deshalb in guter Näherung annehmen, dass ein Kettenglied in geraden Strecken einem einachsigen Spannungszustand unterworfen ist.

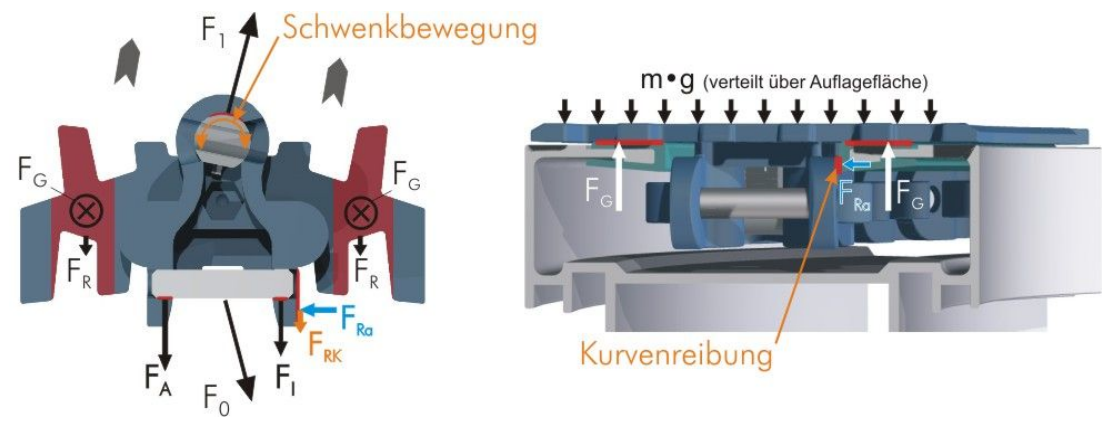

Abb. 3: Belastungsmodell horizontale Kurve

Im Kräftemodell für horizontale Kurven besteht ein wesentlicher Unterschied dazu: die Zugkräfte an beiden Seiten eines Kettengliedes $F_{1}$ und $F_{0}$ liegen nicht auf einer Wirkungslinie, sondern sind um einen bestimmten Winkel zueinander geneigt. Um diesen Winkel müssen sich die Kettenglieder seitlich verdrehen, damit sie die Kurve durchlaufen können. Die resultierende radiale Kraftkomponente $F_{R a}$ verursacht eine zusätzliche Kurvenreibkraft $F_{R K}$ an der Kontaktstelle zum Steg der Gleitschiene, wodurch das Kettenglied asymmetrisch belastet wird.

Eine noch stärkere asymmetrische Belastung tritt an den Übergangsstellen zwischen Geraden und Kurven auf. Bei der seitlichen Schwenkbewegung des jeweils vorhergehenden Kettengliedes in die Kurve hinein und wieder heraus wird der Pin, der im vorderen Teil des Kettengliedes (Auge) gleitend gelagert ist, unter voller Belastung durch die Kettenzugkraft verdreht. Dadurch tritt 
kurzzeitig ein Reibmoment bzw. eine Reibkraft am Kettenauge auf, welches dadurch in Richtung Kurvenmittelpunkt (Kurvenanfange) bzw. entgegengesetzt gedrückt wird. Diese Schwenkkraft leitet sich über den Reibwert zwischen Pin und Kette direkt aus der Kettenzugkraft ab und erreicht dadurch eine nicht zu vernachlässigende Höhe. In Bezug auf den Beanspruchungszustand bedeutet dies, dass ein Kettenglied beim Durchlaufen von Kurvenstrecken, v.a. aber an den Übergängen zwischen Geraden und Kurven einer mehrachsigen Beanspruchung unterliegt.

\section{FEM-Analyse der Beanspruchungen}

Für die aufgestellten Belastungsmodelle wurden Untersuchungen mit der Finite Elemente Methode (FEM) durchgeführt, um Verteilung und Maximalwert der im Kettenglied hervorgerufenen Spannungen zu ermitteln. Zur genaueren Modellierung der Kraftübertragung auf das Kettenglied wurden Pin, Bolzen und Gleitschiene über Kontaktbedingungen in die Berechnung einbezogen. Die Belastung wurde aufgebracht, in dem der Bolzen als raumfest definiert und am Pin eine Zugkraft von $1.200 \mathrm{~N}$ aufgebracht wurde. Die im Vergleich zur Kettenzugkraft relativ kleinen Reibkräfte wurden bei der Simulation vernachlässigt.

Beim Vergleich der errechneten Spannungsverteilungen für gerade Strecken, Kurven sowie den Übergang zwischen beiden werden deutliche Unterschiede sichtbar (Abbildung 4). Im Falle des einachsigen Zuges, der die Belastung in geraden Strecken repräsentiert, stellt sich eine symmetrische Deformation und Spannungsverteilung ein. In der Kurve verformt sich der vordere Teil des Kettengliedes aufgrund der radialen Komponente der schräg angreifenden Zugkraft am Pin in Richtung Kurvenmittelpunkt. Die kurvenabgewandte Lasche wird verstärkt belastet.

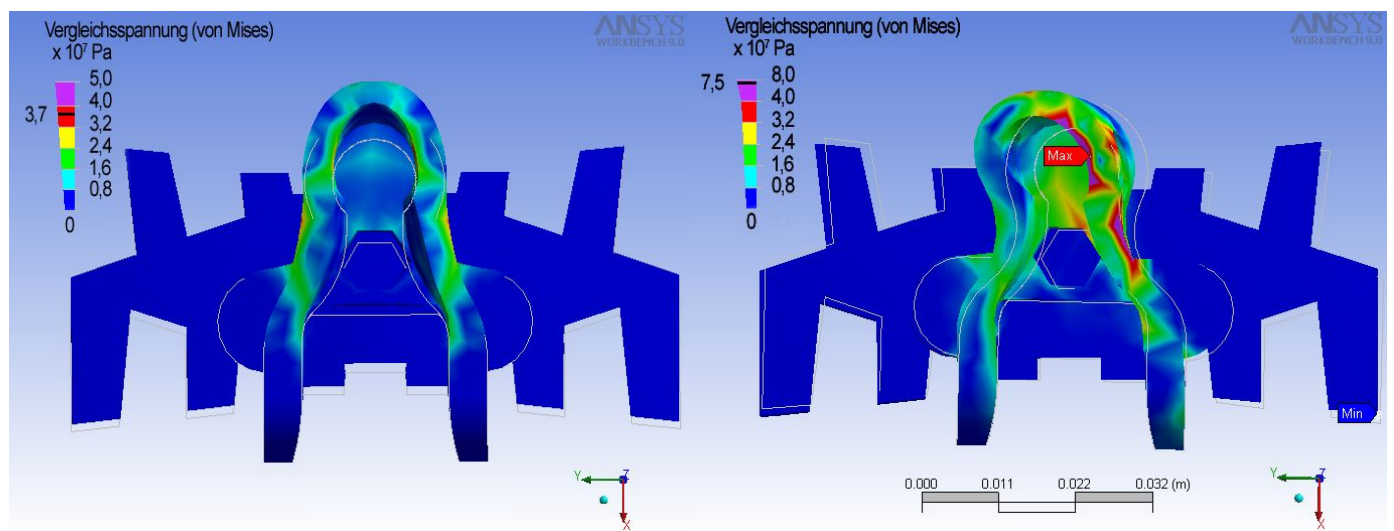

Abb. 4: Van-Mises-Vergleichsspannung (1. Gerade, r. Übergang zwischen Gerade und Kurve)

Beim Einschwenken des Pins, das durch eine in Richtung Kurvenmitte weisende, zuätzliche Reibkraft am Kettenauge modelliert wurde, wird die Außenlasche nochmals wesentlich stärker beansprucht. Dabei treten nicht nur höhere Maximalwerte der Vergleichsspannung auf, sondern auch die Größe hochbelasteter Gebiete im Bereich um die Aussparung für den Bolzen am Kettenauge nimmt zu.

Die Ergebnisse der Simulation unterstreichen, dass ein Kettenglied bei gleicher Kettenzugkraft in Kurven, besonders aber an den Übergängen zwischen Kurven und Geraden, stärker beansprucht wird als in einer Geraden. Im Unterschied zur Simulation ist die Belastung der Kette beim Durchlaufen einer Förderstrecke nicht statisch, sondern durch einen zeitabhängigen Verlauf gekennzeichnet. Dabei entspricht jeder Umlauf einem schwellenden Lastwechsel, der die Kette schädigt. Befinden sich Kurven in der Förderstrecke, wird die Kette durch die erhöhte Beanspruchung bei gleicher Zugkraftamplitude stärker geschädigt, als in einer ausschließlich geraden Strecke. Die Festigkeitsreserven der Kette werden eher aufgebraucht, so dass die Lebensdauer der wesentlich reduziert wird. Die in [Auerbach06] dargestellten Ergebnisse von Dauerfestigkeitsuntersuchungen auf Testförderstrecken bestätigen diese Schlussfolgerung. 


\section{Untersuchung der Kettenzugkraft}

Um den bereits angesprochenen zeitlichen Verlauf der Kettenzugkraft während des Kettenumlaufes näher untersuchen zu können, musste ein geeignetes System zur Messung und Übertragung der Kettenzugkraft bei laufender Kette entwickelt werden. Dessen wichtigste Komponente ist das Messkettenglied, das durch Modifikation eines Standardkettengliedes angefertigt wurde. Dazu wurde der Stahlbolzen hohlgebohrt, an zwei gegenüberliegenden Seiten angeschliffen und mit Dehnmessstreifen versehen. Das Kettenglied wurde mit einem speziellen Pin sowie einer Bohrung zum Herausführen der Anschlussleitungen versehen. Anschließend erfolgte die Entwicklung der Elektronik zur Bereitstellung der Brückenspeisespannung sowie der Digitalisierung und drahtlosen Übermittlung der Messwerte zu einem Messrechner. Der Sender konnte in ein sehr kompaktes Gehäuse integriert werden, dass auf der Kette montiert werden und mit dieser durch die gesamte Förderstrecke umlaufen konnte. Mit diesem Meßsystem (Abbildung 5) wurden Förderstrecken, die in wechselnden Kombinationen alle bekannten Komponenten zur Streckenführung (Gerade, Bogenrad, horizontaler und vertikaler Gleitbogen, Antrieb und Umlenkung) enthielten, bei verschiedenen Fördergeschwindigkeiten und Belastungen durch Fördergut untersucht.

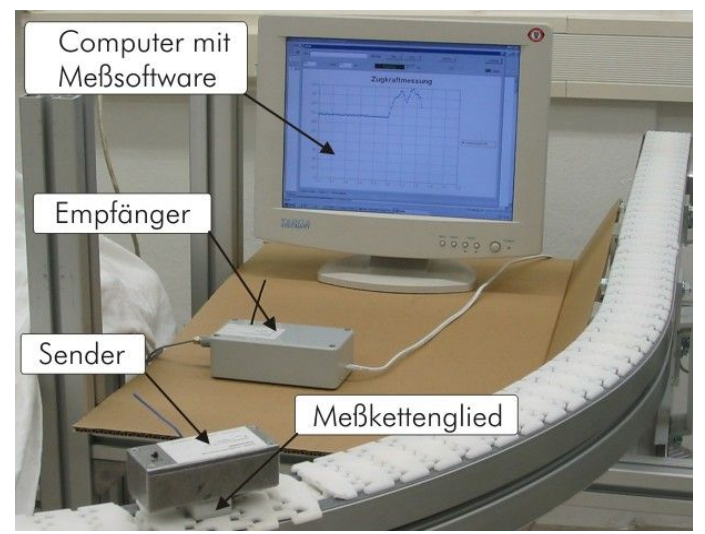

Abb. 5: System zur Zugkraftmessung

Dabei wurde deutlich, dass die Kettenzugkraft bei einem kompletten Umlauf durch eine Förderstrecke folgenden grundsätzlichen Verlauf aufweist: Sie wächst vom kraftfreien Zustand im Kettensack des Antriebs während des weiteren Weges durch das Führungssystem über Untertrum, Umlenkung und Obertrum immer weiter an und erreicht beim Eingriff des Antriebsritzels ihren Maximalwert. Abbildung 6 zeigt dies am Beispiel einer Förderstrecke, die aus einem $90^{\circ}$ Gleitbogen zwischen zwei geraden Strecken bestand.

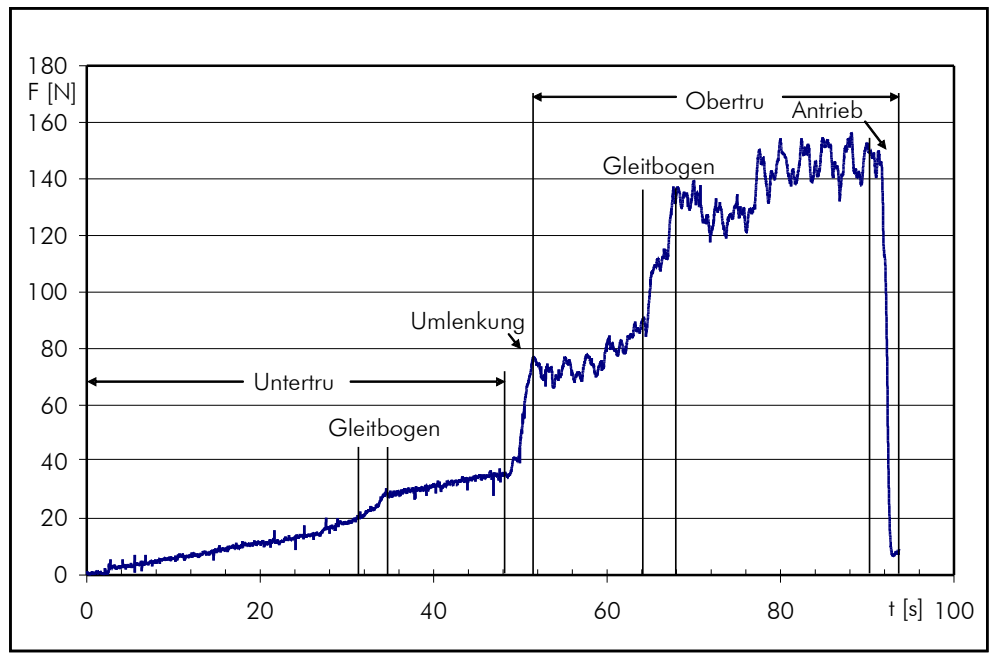

Abb. 6: Kettenzugkraft bei einem kompletten Umlauf

Im dargestellten Zugkraftverlauf lassen sich eindeutig Abschnitte mit unterschiedlichem Anstieg erkennen und den verschiedenen Streckenabschnitten zuordnen. Die geraden Strecken bewirken 
einen linearen Zugkraftanstieg, während der Gleitbogen einen überproportionalen Zuwachs hervorruft. Mit der Annäherung an den Antrieb überlagern zunehmend Schwingungen den Kraftverlauf, die durch den Polygoneffekt des Antriebsritzels hervorgerufen werden.

Im Folgenden wird kurz auf die Besonderheiten einzelner Streckenelemente sowie den Einfluss von Reibwert und Geschwindigkeit auf den Zugkraftverlauf eingegangen.

\subsection{Gerade Strecken}

Der bereits erwähnte lineare Kraftanstieg in geraden Strecken wird von den spezifischen Massen von Kette und Fördergut sowie vom Reibwert zwischen Kette und Gleitschiene bestimmt. Ausschlaggebend für den weiteren Verlauf ab einem betrachteten Punkt ist dabei der Zuwachs an insgesamt transportierter Masse, wie die Kurven der drei Lastfälle Leerlauf, Staubetrieb und Guttransport in Abbildung 7 verdeutlichen.

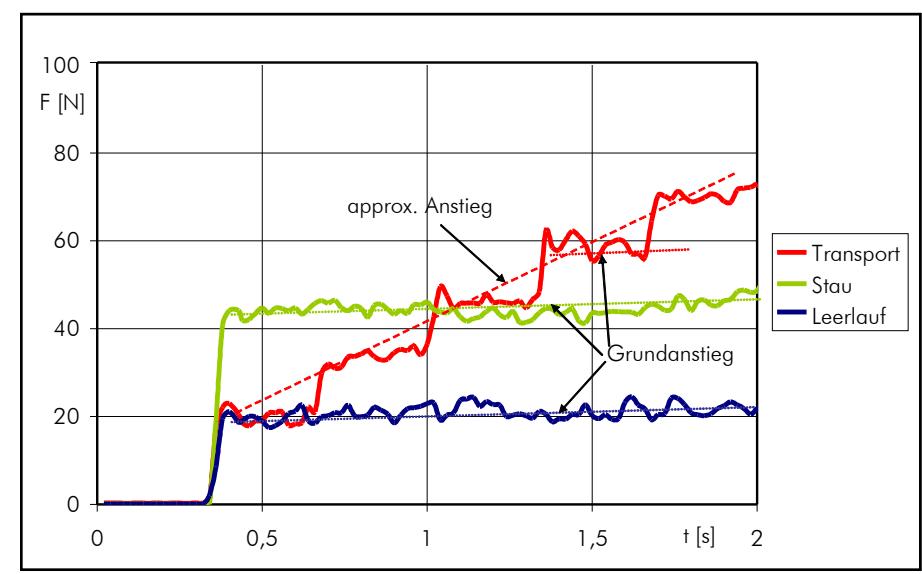

Abb. 7: Zugkraftverlauf gerade Strecke

Sowohl im Leerlauf als auch im Staubetrieb kommt kein weiteres Fördergut hinzu, so dass die Kettenzugkraft mit einem geringen Grundanstieg weiter-wächst, der von der Eigenmasse der Kette bestimmt wird. Dieser Grundanstieg wird jedoch nicht von der Höhe der bereits vorhandenen Kettenzugkraft beeinflusst. Legt man zur Simulation des Transportes von Fördergut in konstanten Intervallen Massestücke auf die laufende Kette, ergibt sich der gestufte Verlauf der roten Kurve in Abbildung 7. Würde man immer kleinere Massestücken in kürzeren Abständen auflegen, ergäbe sich wieder ein linearer Verlauf, der allerdings einen größeren Anstieg als ohne neu hinzukommendes Gutmassen aufweist.

\subsection{Horizontaler Gleitbogen}

Der Zugkraftverlauf in horizontalen Gleitbögen weist einen exponentiellen Anstieg auf. Der Kraftzuwachs ist somit nicht nur von der hinzukommenden Masse, sondern auch von der Kettenzugkraft am Anfang der Kurve abhängig. Dies wird deutlich, wenn man die Kurven für Leerlauf und Staubetrieb in Abb. 8 vergleicht.

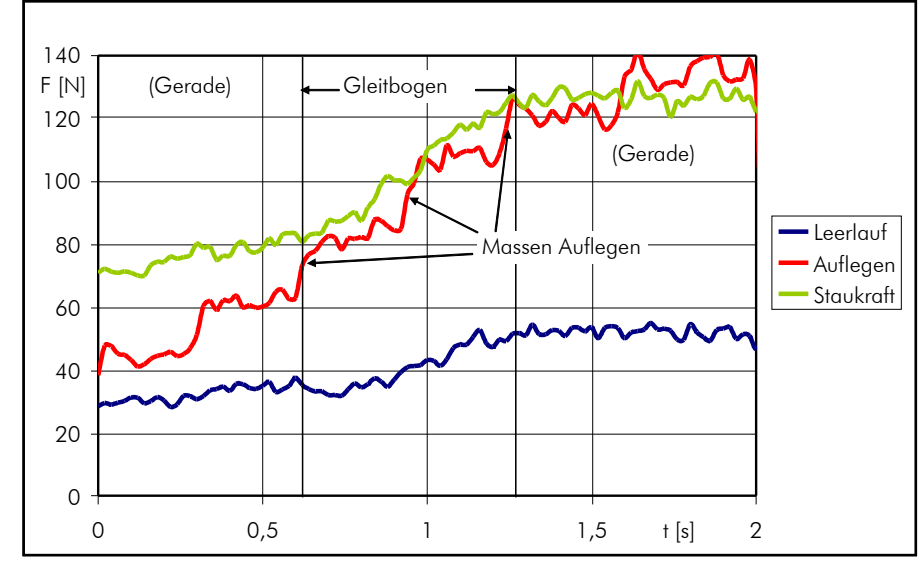

Abbildung 8: Zugkraftverlauf horizontaler Gleitbogen 
In beiden Fällen wird nach Beginn der Messung kein weiteres Fördergut aufgebracht, aber die höhere Anfangszugkraft infolge des Staubetriebs führt zu einem steileren Anstieg des weiteren Kraftverlaufes sowie zu einer insgesamt größeren Kraftzunahme. Beim Auflegen von Fördergut sind wieder Sprünge zu verzeichnen, die gemittelt einen stärkeren (exponentiellen) Anstieg, als bei leerlaufender Kette ergeben.

\subsection{Bogenräder}

Bogenräder werden vom Messkettenglied sehr schnell durchlaufen, da diese relativ kleine Radien bzw. Umfänge besitzen. Auch bei geringer Fördergeschwindigkeit von z.B. $5 \mathrm{~m} / \mathrm{min}$ reicht die zur Verfügung stehende Zeitspanne nicht aus, um mehrere Gutstücke aufzulegen, so dass auf eine Simulation des Guttransportes verzichtet werden musste. Statt dessen wurde der Kraftverlauf bei mehreren Anfangszugkräften aufgezeichnet, wie Abb. 9 zeigt.

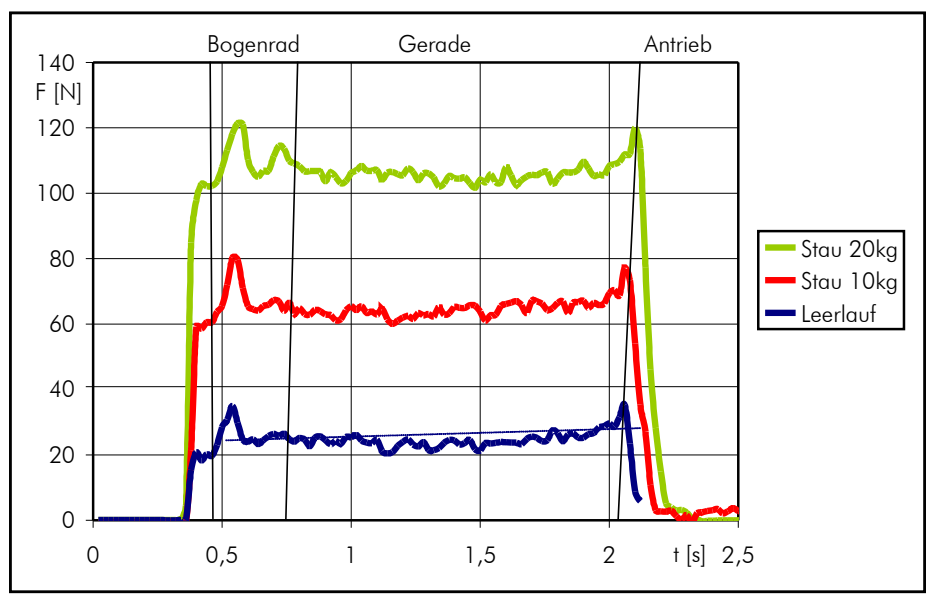

Abbildung 9: Zugkraftverlauf Bogenrad

Im Diagramm fallen Kraftspitzen am Anfang und am Ende des Bogenrades auf - sie sind ein Hinweis auf die mehrachsige Beanspruchung, die durch die Reibkräfte zwischen Pin und Kettenauge beim seitlichen Ein- und Ausschwenken verursacht wird (vgl. Abschn. Belastungsmodell). Nach Verlassen der Kurve hat die Kettenzugkraft jedoch nicht mehr zugenommen, als dies in einer geraden Strecke der Fall wäre. Ebenso ist die Kraftzunahme in Bogenrädern nicht von der Anfangszugkraft abhängig.

\subsection{Einfluss der Fördergeschwindigkeit}

Im untersuchten Geschwindigkeitsbereich von 5-40 m/min konnte kein signifikanter Einfluss der Fördergeschwindigkeit auf den Zugkraftverlauf festgestellt werden. Die Reibwerte der untersuchten Materialpaarungen (Delrin/PE UHMW und Delrin PVDF) sind in diesem Bereich offenkundig weitgehend konstant. Die bei verschiedenen Geschwindigkeiten ermittelten Kraftverläufe sind bei ansonsten gleichen Bedingungen nur unterschiedlich zeitlich skaliert und könnten durch Stauchen bzw. Dehnen zur Deckung gebracht werden.

\subsection{Einfluss des Reibwertes}

Am deutlichsten zeigt sich der Einfluss des Reibwertes in Gleitbögen, weil dort zusätzlich die Kurvenreibung auftritt. Untersucht wurden die Paarungen Delrin-Kette gegen Gleitleisten aus PEUHMW sowie PVDF, für die Reibwerte von 0,26 und 0,15 ermittelt wurden (Abb. 10).

Der höhere Reibwert der PE-Schiene führt erwartungsgemäß zu einer stärkeren Kraftzunahme, die darüber hinaus von der Anfangszugkraft abhängt. Bei der Anfangszugkraft von $300 \mathrm{~N}$ ist die Kraftzunahme durch den höheren Reibwert deutlich größer als bei 100 N. Dies trifft aber nur auf Gleitbögen $\mathrm{zu}$ - in geraden Strecken ist die Kraftzunahme unabhängig von der Anfangskraft. Höhere Reibwerte bewirken hier nur einen größeren linearen Anstieg. 


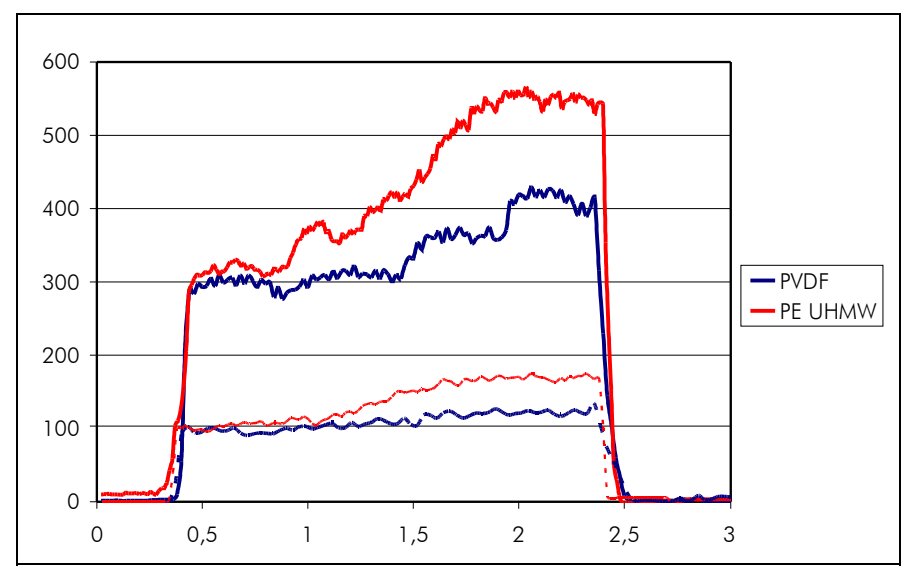

Abbildung 10: Einfluss des Reibwertes

\section{Zugkraftberechnung in Gleitbögen}

Die unterschiedlichen Kraftverläufe in geraden Strecken und Kurven erfordern es, GleitkettenFördersysteme zur Berechnung der Zugkraft in entsprechende Abschnitte einzuteilen, für die jeweils andere Gleichungen gelten. Die Berechnung wird unmittelbar nach dem Antrieb im Untertrum (Zugkraft praktisch null) begonnen und über Umlenkung und Obertrum bis zum Antrieb fortgeführt, bei dem die maximale Zugkraft erreicht wird. Der Index $k$ bezeichnet die Nummer des gerade berechneten Abschnittes, $F_{k}$ entsprechend die an dessen Ende erreichte Zugkraft.

Der lineare Kraftanstieg in geraden Strecken und Bogenrädern wird durch die bekannte Gleichung:

$$
F_{k}=F_{k-1}+L \cdot \mu \cdot g \cdot\left(q_{c}+q_{p}\right)
$$

bereits zutreffend beschrieben. Da sich in Gleitbögen die Reibkräfte durch die Masse von Gut und Kette und die Kurvenreibung gegenseitig beeinflussen, ist die exakte Berechnung der Kettenzugkraft bislang nicht ohne weiteres möglich gewesen [Monsberger79]. Es werden deshalb sog. Bogenfaktoren $\mathrm{K}_{\alpha}$ verwendet (z.B. in [Rexnord00], [FlexLink99] und [Flexon03]), mit denen der Kraftzuwachs durch Gleitbögen näherungsweise berechnet werden kann:

$$
F_{k}=\left[F_{k-1}+L \cdot \mu \cdot g \cdot\left(q_{c}+q_{p}\right)\right] \cdot K_{\alpha}
$$

Häufig werden diese tatsächlich nur für einen bestimmten Reibwert zwischen Kette und Gleitschiene gültigen Faktoren pauschal für alle Materialpaarungen angegeben, so dass je nach dem tatsächlich vorhandenen Reibwert ein mehr oder weniger großer Fehler der Zugkraft resultiert. Unberücksichtigt bleibt dabei weiterhin der Einfluss des Kurvenradius, der v.a. bei hohen spezifischen Gutmassen nennenswerte zusätzliche Zugkräfte verursachen kann. Um diese Probleme zu beheben, wurden ausgehend vom Kräftegleichgewicht an einem (infinitesimalen) Kettenstück (Abb. 11) neue Gleichungen für horizontale und vertikale Gleitbögen hergleitet.
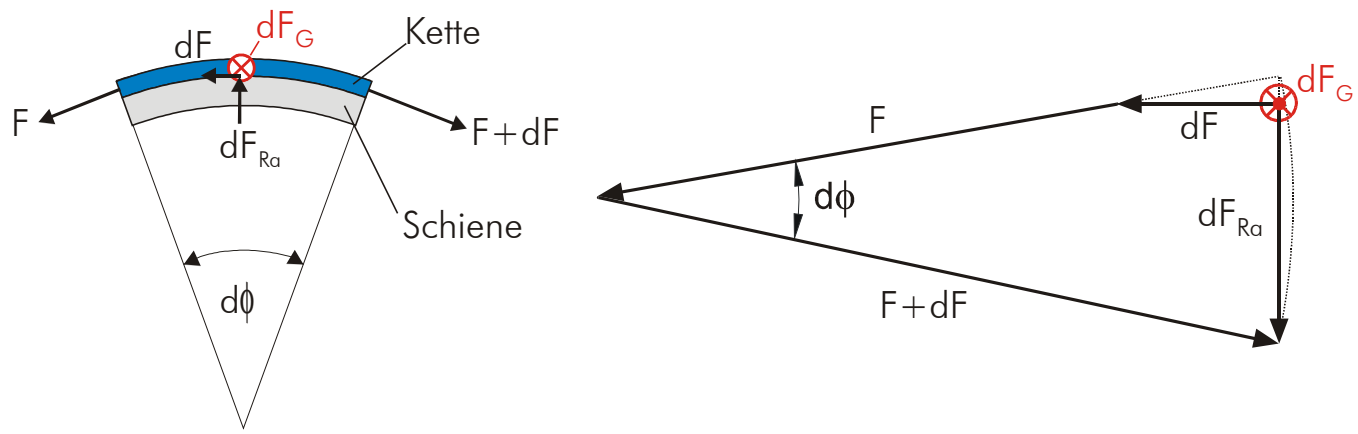

Abbildung 11: Kräfte am Kurvenstück, Kräfteplan

Am Beispiel des horizontalen Gleitbogens soll nun die Herleitung kurz dargestellt werden. Die Änderung der Zugkraft $d F$ ergibt sich aus Radial- und Gewichtskraftanteilen und dem Reibwert $\mu$ :

$$
d F=\mu \cdot\left(d F_{R a}+d F_{G}\right)
$$


Um die Radialkraft zu berechnen, wurden vereinfacht $\sin d \phi \approx d \phi$ und $d F \cdot d \phi \approx 0$ gesetzt, was für infinitesimale $d \phi$ zulässig ist. Die Radialkraft $d F_{R a}$ erhält man dann durch:

$$
d F_{R a}=F \cdot d \phi
$$

Die Gewichtskraft $d F_{G}$ ergibt sich aus der Bogenlänge und der längenbezogenen Gewichtskraft $q=g \cdot\left(q_{c}+q_{p}\right)$ von Gut und Kette:

$$
d F_{G}=r \cdot q \cdot d \phi
$$

Nach Einsetzen von (3) und (4) in (5) und Umstellen erhält man:

$$
\frac{d F}{F+r \cdot q}=\mu \cdot d \phi
$$

Gl. (6) lässt sich unter Verwendung folgender Beziehung integrieren:

$$
\int \frac{d x}{x+a}=\ln (x+a)
$$

Integriert man beide Seiten in Gl.(6), ergibt sich damit:

$$
\int_{F\left(\phi_{0}\right)}^{F(\phi)} \frac{d F}{F+r \cdot q}=|\ln (F+r \cdot q)|_{F\left(\phi_{0}\right)}^{F(\phi)} \quad \text { und } \int \mu d \phi=\mu \cdot \phi
$$

Nach Einsetzen der Grenzen und Potenzieren beider Seiten zur Basis e entsteht:

$$
\frac{F(\phi)+r \cdot q}{F\left(\phi_{0}\right)+r \cdot q}=e^{\mu \phi}
$$

Führt man zur Kennzeichnung der Abschnittsgrenzen den Abschnittsindex $k$ ein, d.h.:

$$
F(\phi)=F_{k} \text { und } F\left(\phi_{0}\right)=F_{k-1}
$$

und stellt nach der Zugkraft am Ende des Abschnitts $F_{k}$ um, erhält man:

$$
F_{k}=\left(F_{k-1}+r \cdot q\right) \cdot e^{\mu \phi}-r \cdot q
$$

bzw. nach Einsetzen von $q=g \cdot\left(q_{c}+q_{p}\right)$ :

$$
F_{k}=\left[F_{k-1}+r \cdot g \cdot\left(q_{c}+q_{p}\right)\right] \cdot e^{\mu \phi}-r \cdot g \cdot\left(q_{c}+q_{p}\right)
$$

Mit Gl. (14) kann die Zugkrafterhöhung durch horizontale Gleitbögen für beliebige Anfangskräfte $F_{k-1}$, Reibwerte $\mu$ und Kurvenradien $r$ exakt berechnet werden. Der Vergleich mit gemessenen Kraftverläufen (Abb. 12) ergab eine gute Übereinstimmung mit nach Gl. (14) berechneten.

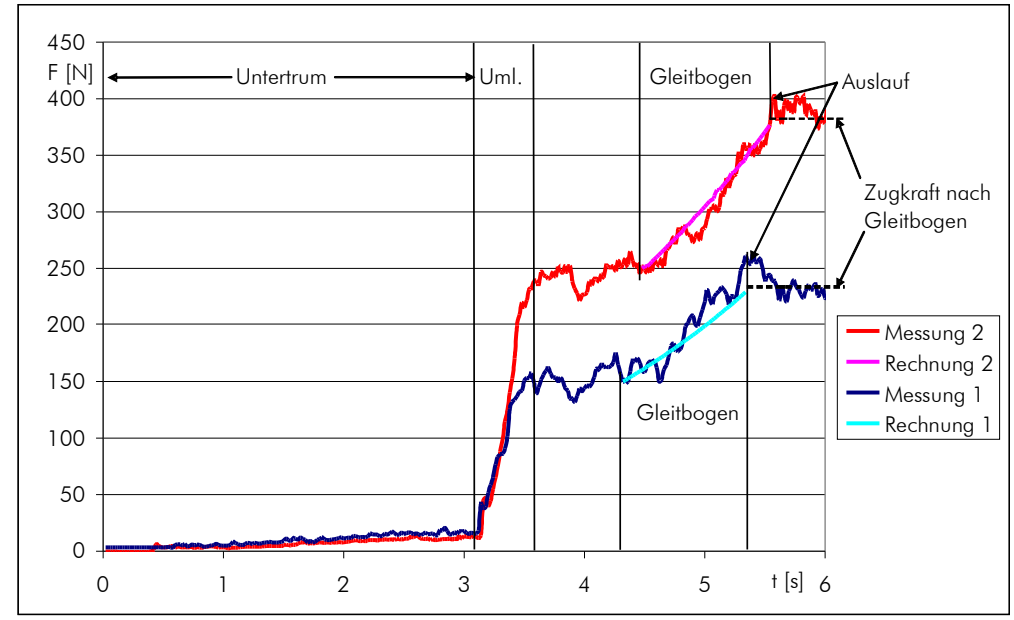

Abbildung 12: Vergleich Messung / Rechnung 
Für vertikale Gleitbögen wurde in ähnlicher Weise eine neue Gleichung abgeleitet:

$$
F_{k}=\left[\frac{F_{k-1}-r \cdot g \cdot\left(q_{c}+q_{p}\right) \cdot\left(\sin \phi_{0}-\mu \cdot \cos \phi_{0}\right)}{e^{\mu \phi_{0}}}\right] \cdot e^{\mu \phi}-r \cdot g \cdot\left(q_{c}+q_{p}\right) \cdot(\sin \phi-\mu \cos \phi)
$$

Auf eine Darstellung der Herleitung wird aus Platzgründen verzichtet. Die gezeigten Gleichungen sind auf weitere Förderer übertragbar, die ein in ähnlicher Weise von Kurvenreibkräften betroffenes, massebeaufschlagtes Zugmittel verwenden (z.B. Mattenketten, Rundriemen, Gurte, Seile mit Tragelementen o. ä.). Dies konnte auch durch einen Vergleich mit den in [Krause99] dargestellten Berechnungsgleichungen für Stauscheibenförderer gezeigt werden, die sich auf den allgemeinsten Fall eines beliebig im Raum geneigten Bogens beziehen.

\section{Zusammenfassung und Ausblick}

Im Ergebniss der Untersuchungen ist festzustellen, dass Zugkraftentwicklung und Beanspruchung seitenbeweglicher Gleitketten stark vom Aufbau der Förderstrecke abhängig sind. Dabei muss vor allem zwischen einer einachsigen Zugbeanspruchung in geraden Strecken und einer mehrachsigen, erhöhten Beanspruchung in horizontalen Kurven unterschieden werden. Es wurden Gleichungen vorgestellt, die eine exaktere Berechnung des Zugkraftverlaufs in Gleitbögen als die bislang üblichen Bogenfaktoren ermöglichen und sich auf ähnlich geartete Zugmittel anderer Fördersysteme übertragen lassen. Im Gegensatz zu den Bogenfaktoren können damit alle relevanten Parameter, wie Reibwert, Kurvenradius und längenbezogene Massen, korrekt berücksichtigt werden. Es konnte gezeigt werden, dass sich gemessene Kraftverläufe in Gleitbögen gut mit den neuen Gleichungen beschreiben lassen.

In [Auerbach06] werden die Auswirkungen der verschiedenen Belastungsfälle auf die Lebensdauer der Ketten dargestellt. Dazu werden vergleichende Versuche auf einer dynamischen Werkstoffprüfmaschine sowie auf Testförderstrecken mit unterschiedlicher Streckenführung beschrieben, die Rückschlüsse auf den Einfluss einzelner Streckenelemente auf die (bruchbedingte) Lebensdauer der Gleitketten erlauben. Weiterhin wird ein Ansatz vorgeschlagen, der erstmals eine rechnerische Abschätzung der Lebensdauer der Gleitketten ermöglicht und eine darauf basierende Software vorgestellt.

\section{Formelzeichen}

$\begin{array}{ccl}\text { Zeichen } & \text { Einheit } & \text { Beschreibung } \\ F_{0} & \mathrm{~N} & \text { Kraft am Anfang eines Kettengliedes } \\ F_{1} & \mathrm{~N} & \text { Kraft am Ende eines Kettengliedes } \\ F_{G} & \mathrm{~N} & \text { Gewichtskraft von Kette und Gut } \\ F_{k} & \mathrm{~N} & \text { Kettenzugkraft am Ende von Abschnitt } \mathrm{k} \\ F_{R a} & \mathrm{~N} & \text { Radialkraft } \\ F_{R K} & \mathrm{~N} & \text { Kurvenreibungskraft } \\ g & \mathrm{~m} / \mathrm{s}^{2} & \text { Graviationskonstante } \\ k & - & \text { Nummer des berechneten Abschnittes } \\ K_{\alpha} & - & \text { Bogenfaktor } \\ L & \mathrm{~m} & \text { Länge des Abschnittes } \\ q_{c} & \mathrm{~kg} / \mathrm{m} & \text { Längenbezogene Masse der Kette } \\ q_{p} & \mathrm{~kg} / \mathrm{m} & \text { Längenbezogene Masse des Fördergutes } \\ q & \mathrm{~N} / \mathrm{m} & \text { Längenbezogene Gewichtskraft von Fördergut und Kette } \\ r & \mathrm{~m} & \text { Kurvenradius } \\ \phi & - & \text { Umlenkwinkel einer Kurve } \\ \phi_{0} & - & \text { Steigungswinkel am Anfang einer vertikalen Kurve } \\ \mu & - & \text { Gleitreibungskoeffizient zwischen Kette und Gleitschiene }\end{array}$


DOI: 10.2195/LJ_Ref_D_Auerbach_0920062

\section{Literatur}

[Bosch04] Firmenschrift Bosch, VarioFlow Kettenfördersystem, 2004

[Auerbach06] Auerbach, P.; Nendel, K., Untersuchung der Beanspruchung und des Zugkraftverlaufs raumgängiger Gleitketten, 2006

[Monsberger79] Monsberger, Josef: Tragkettenförderer sind vereinfachte Bauformen von Plattenbandförderern. In: Maschinenmarkt 65 (1979), S. 1272

[Rexnord00] Firmenschrift Rexnord, Konstruktionsanleitung Scharnier- und Plattenbandketten, 2000

[FlexLink99] Firmenschrift FlexLink, Modulares System XT, 1999

[Flexon03] Firmenschrift Flexon, Produktprogramm Stahl- und Kunststoffketten, 2003

[Krause99] Krause, F. et.al.: Theoretische und experimentelle Untersuchungen an Stauscheibenförderern (Rohrkettenförderern). In: Schüttgut 2 (1999), S. 139-149 